\title{
Measurement of the Thermal Absorption Cross Section in Lucite Using Fermi Age Theory
}

\author{
Rene G. Sanchez, David K. Hayes, Travis J. Grove, Jesson D. Hutchinson, Jennifer J. Ong, \\ Fredrik K. Tovesson
}

\begin{abstract}
The diffusion processes of neutrons in Lucite have been investigated. The mechanisms of these processes have been described as having two steps. In the first step, the fast neutrons collide with the nuclei of Lucite, losing energy until they reach thermal energies, In the second step of the diffusion process, the thermal neutrons continue to diffuse through the Lucite without any significant loss of energy until they are finally absorbed or leak out of the system. Experiments were performed to study these two processes and to estimate the absorption cross section in Lucite. The experiments yielded an average range of $6.3 \mathrm{~cm}$ for fast neutrons slowing down to thermal energies and a thermal absorption cross section in Lucite of 0.52 b.
\end{abstract}

Index Terms-activation, absorption cross section, fast neutrons, thermal energies, thermal neutrons, diffusion process.

\section{INTRODUCTION}

$I^{1}$ is well known that when fast neutrons are emitted by a neutron source inside a hydrogenous material, two essentially distinct diffusion processes take place. In the first diffusion process, known as the slowing-down phase, the fast neutrons from the neutron source collide with the nuclei of the moderating material, losing energy until they reach thermal energies $(0.052 \mathrm{eV})$. In the second diffusion process, the thermal neutrons continue to diffuse throughout the moderating material without significant energy loss, when compared with the energy loss during the slowing-down phase, until they are finally absorbed. The second diffusion process is also known as the thermal diffusion phase. In this process, the larger the number of collisions, the lower the neutron energy will be. Neutrons that have undergone more collisions, on the average, will have traveled larger distances from the neutron

Manuscript received January 18, 2009. This work was supported in part by the U.S. Department of Energy

R. G. Sanchez is with Los Alamos National Laboratory, Los Alamos, NM 87545 USA (phone: 505-665-5343; fax: 505-665-9849; e-mail: rsanchez@, lanl.gov)

D. K. Hayes is with Los Alamos National Laboratory, Los Alamos, NM 87545 USA (e-mail: dkhayes@lanl.ogv).

T. J. Groveis is with Los Alamos National Laboratory, Los Alamos, NM 87545 USA (e-mail: tgrove@lanl.gov).

J. D. Hutchinson is with Los Alamos National Laboratory, Los Alamos, NM 87545 USA (e-mail: jesson@lanl.gov).

J. J. Ong is with Los Alamos National Laboratory, Los Alamos, NM 87545 USA (e-mail: jiong@lanl.gov)

F. K. Tovesson is with Los Alamos National Laboratory, Los Alamos, NM 87545 USA (e-mail: tovesson@lanl.gov)

U.S. Government work not protected by U.S. copyright source. It is important to point out that the diffusion length during the slowing-down phase depends on the number of collisions required for reducing the initial energy of the neutrons to thermal energy. On the other hand, the diffusion length during the thermal diffusion phase depends on the absorption cross section of the moderating material that is being considered. However, this model is very simplistic because it does not take into account the chemical binding energy of the molecules in the moderating material.

The purpose of this research is to investigate the two diffusion processes in Lucite, obtain a value for its absorption cross section, and compare this value to the one in ENDF/B VI cross-section library. The measurements of these diffusion processes are based on previous measurements that Enrico Fermi performed in the 1930s and 1940s to investigate the neutron absorption properties of various materials, the results of which are documented in Refs. 1 and 2.

\section{MATERIALS AND EQUIPMENT}

\section{A. Lucite Plates and Neutron Source}

The materials used in this experiment consisted of 18 Lucite plates, $35.56 \mathrm{~cm}$ square and $1.18 \mathrm{~cm}$ thick, used as the moderating material. In addition, six 35.56-cm-square and 2.33-cm-thick Lucite plates were used to form the base and top part of the stack. As illustrated in Fig. 1, the Lucite plates were stacked on top of each other to form a rectangular parallelepiped. Some of the thinner Lucite plates had slots where Lucite inserts could be inserted. One of these inserts, the sampler holder insert, had a recess to accommodate a small rhodium foil. Additional Lucite inserts were used to plug the other slots so that there were no voids in the plates. In this way, the rhodium foil on the sample holder insert could be moved axially and radially during the measurements (Fig. 1). Finally, one of the thinner Lucite plates had a central recess to accommodate an $825-\mu \mathrm{Ci}{ }^{252} \mathrm{Cf}$ neutron source. This plate containing the neutron source was placed near the bottom of the stack. In some of the measurements, the rhodium foil was properly screened with cadmium covers. During all the measurements, the stack of Lucite plates was surrounded by a 0.32 -cm-thick layer of cadmium to reduce, as much as possible, the room return component (Fig. 2). 


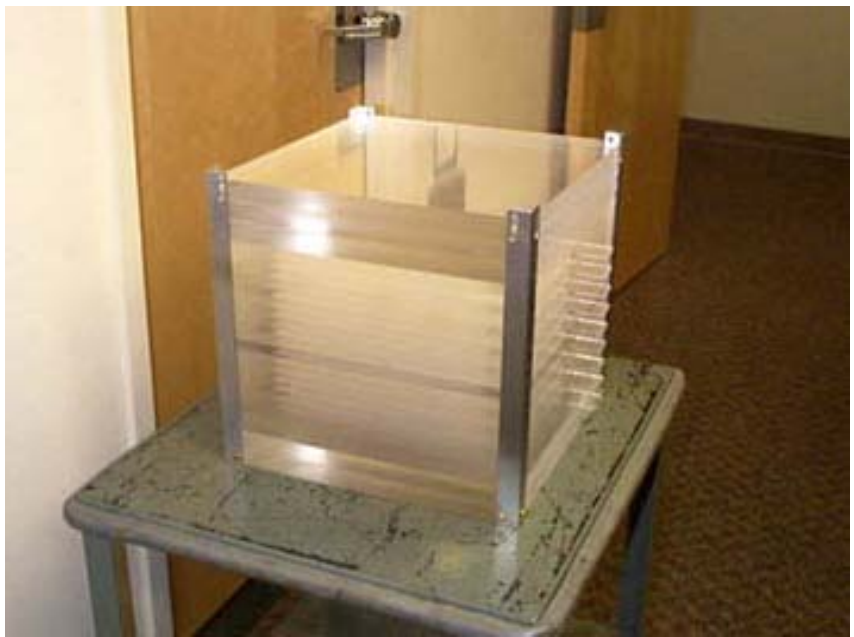

(a)

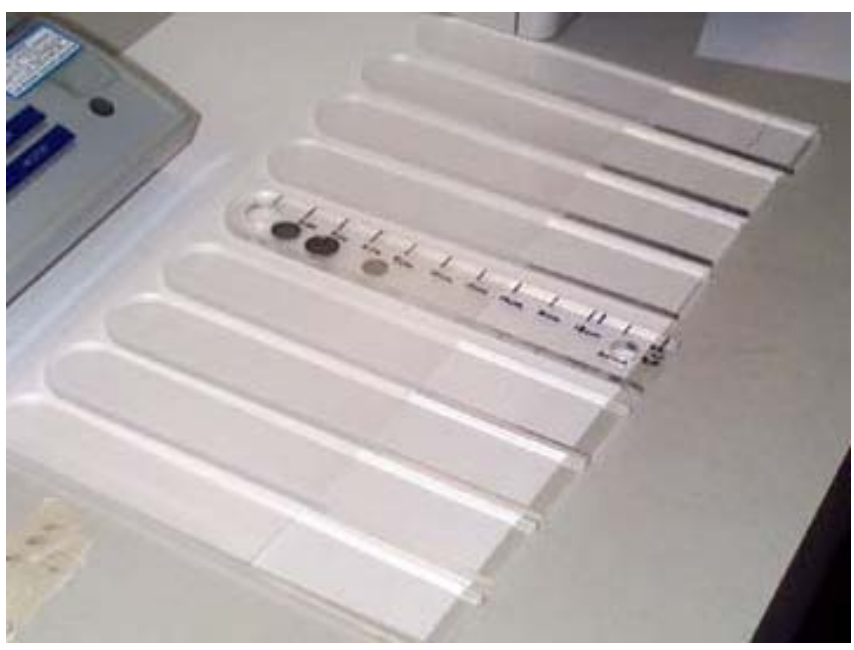

(b)

Fig. 1. (a) Stacking of Lucite plates and (b) rhodium foil and cadmium covers with Lucite inserts.

\section{B. The Rhodium Foil and the Geiger-Muller Counter}

The rhodium foil was $\sim 1.26 \mathrm{~cm}$ in diameter and $0.03 \mathrm{~cm}$ thick. The cadmium covers, which in some cases enclosed the rhodium foil, were $1.69 \mathrm{~cm}$ in diameter and $0.07 \mathrm{~cm}$ thick. The rhodium foil, which is entirely ${ }^{103} \mathrm{Rh}$, is activated by the neutrons emitted from the neutron source through the nuclear reactions shown in Eq. (1):

$$
{ }^{103} \mathrm{Rh}+\mathrm{n} \rightarrow{ }^{104} \mathrm{Rh}
$$

and

$$
{ }^{104} \mathrm{Rh} \stackrel{\beta(99.5 \%)}{\longrightarrow}{ }^{104} \mathrm{Pd} .
$$

As stated above, ${ }^{104} \mathrm{Rh}$ decays by $\beta$-emission $99.5 \%$ of the time, with a half-life of $41.8 \mathrm{~s}$.

To measure the activation of the rhodium foil, we used an Eberline E-600 portable instrument with an Eberline HP-260 pancake Geiger-Muller (GM) detector. The Eberline E-600 is a radiation instrument that can be connected to a variety of detectors for the detection of contamination or radiation (Fig.

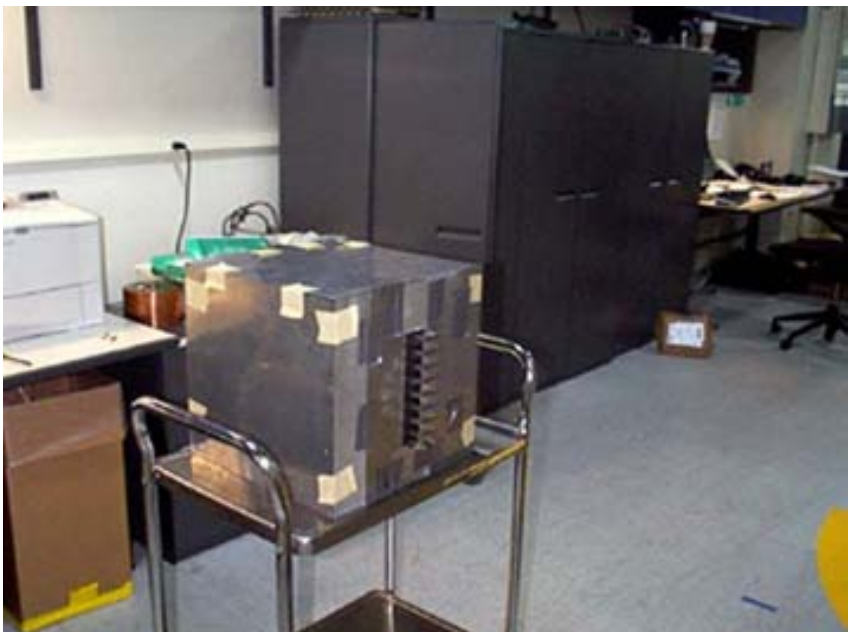

Fig. 2. Experiment setup showing the Lucite stack surrounded by a thin cadmium layer.

3). The Eberline E-600 instrument is capable of measuring alpha, beta, and gamma types of radiation. The E-600 was used as an integrated counter for the $\beta$-measurements described in this paper. The Eberline HP-260 pancake GM detector consists of a small anode plate inside a sealed chamber filled with gas (typically air, 79\% nitrogen). Beta radiation from the activated rhodium foil enters the counting chamber through a window, which is covered with a lowdensity material (mica) to allow the passage of beta particles into the counting region. In the counting region, beta particles interact with the counting gas, producing electron-ion pairs. It is interesting to note that if high voltage applied to the anode is high enough, the electron-ion pairs created initially would be able to trigger many other ion-pair avalanches capable of generating a strong signal that could be easily recorded by the E-600 [3].

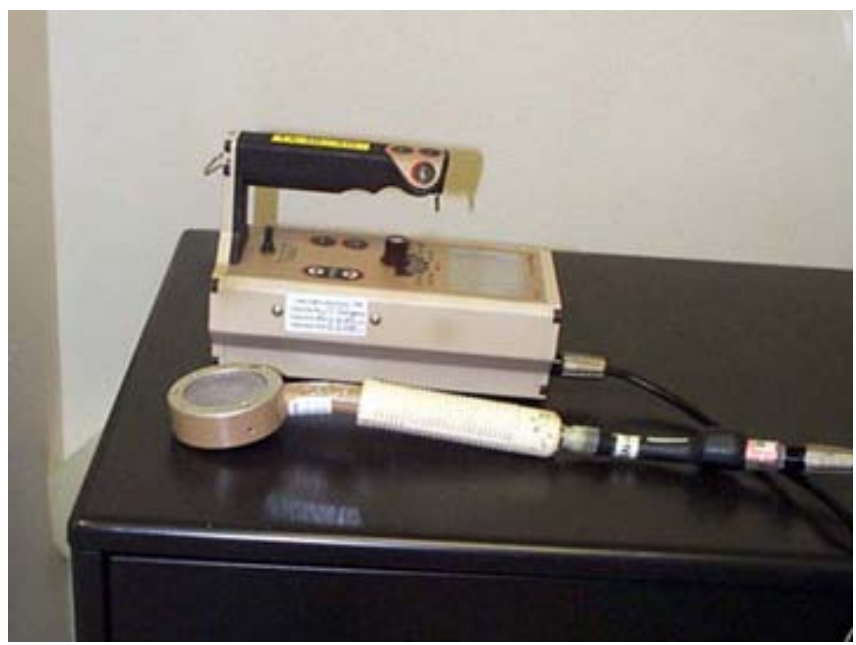

Fig. 3. Eberline E-600 radiation detection instrument with Eberline Hp-260 pancake GM detector.

Once the rhodium foil was irradiated in the Lucite stack at a specific location, the foil was removed from the stack and placed on a cylindrical aluminum block that had recesses for 
the GM detector head and the rhodium foil. This assured that the position of the foil and GM probe were always at the same locations (Fig. 4). Table I shows the dimensions and weights of the materials used in this experiment.

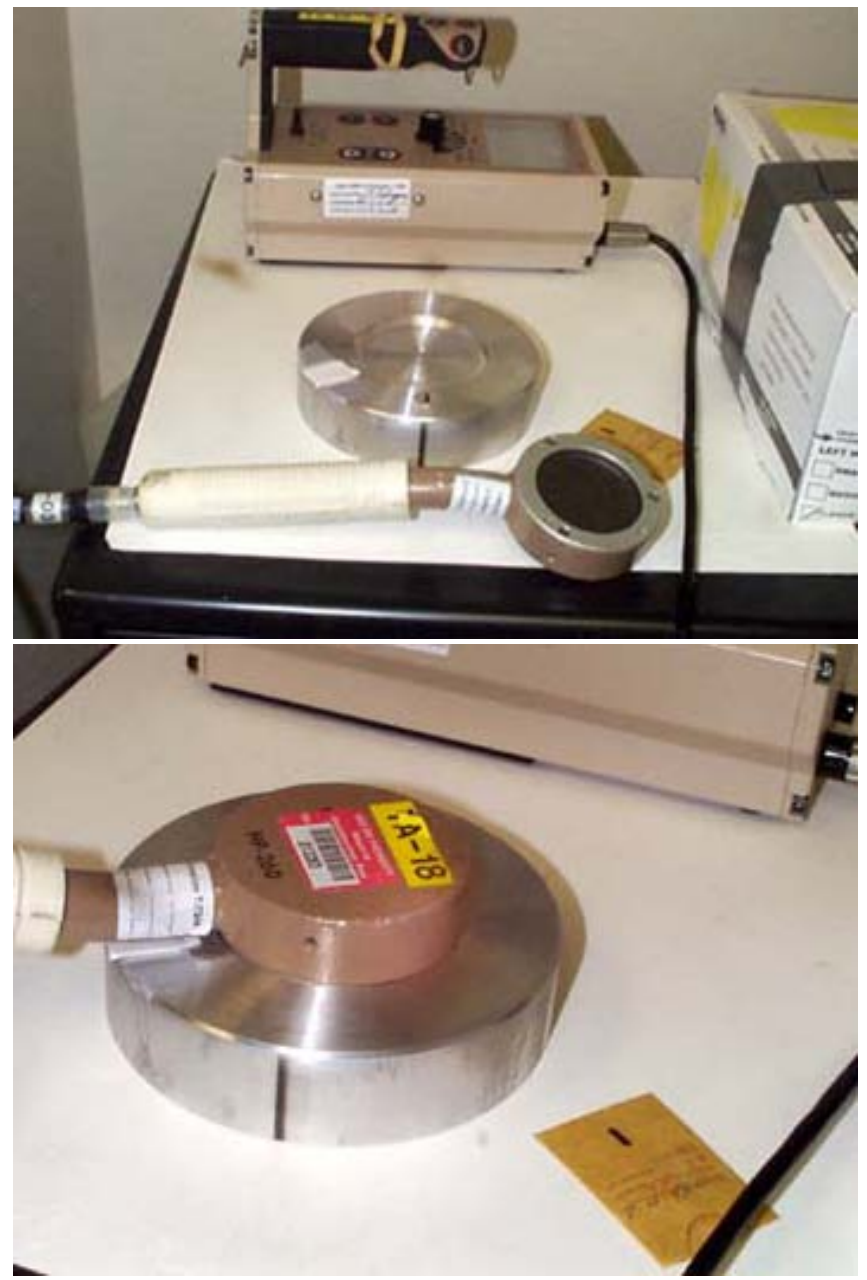

Fig. 4. Aluminum block where rhodium foil and GM probe are placed.

TABLE I

DIMENSIONS AND WEIGHTS OF MATERIALS

\begin{tabular}{|c|c|c|c|}
\hline Material & Dimensions (cm) & Quantity & $\begin{array}{c}\text { Weight } \\
\text { Average } \\
\text { (g) }\end{array}$ \\
\hline $\begin{array}{c}\text { Lucite } \\
\text { (Moderating } \\
\text { Plates) } \\
\text { with Inserts } \\
\end{array}$ & $\begin{array}{c}35.56 \times 35.56 \times \\
1.18\end{array}$ & 18 & 1697.5 \\
\hline $\begin{array}{c}\text { Lucite } \\
\text { (Reflector) }\end{array}$ & $\begin{array}{c}35.56 \times 35.56 \times \\
2.33\end{array}$ & 6 & 3489.2 \\
\hline Rhodium Foil & $\begin{array}{c}1.26 \text { diameter and } \\
0.03 \text { thick }\end{array}$ & 1 & 0.42 \\
\hline $\begin{array}{l}\text { Aluminum } \\
\text { Block }\end{array}$ & $\begin{array}{c}12.7 \text { diameter and } \\
3.37 \text { thick }\end{array}$ & 1 & 1061.7 \\
\hline $\begin{array}{c}252 \mathrm{Cf} \\
\text { Neutron } \\
\text { Source }\end{array}$ & $\begin{array}{c}0.60 \text { diameter and } \\
1.0 \text { height }\end{array}$ & 1 & - \\
\hline
\end{tabular}

\section{SLOWING-Down PROCESS}

We have studied the slowing down of neutrons emitted from ${ }^{252} \mathrm{Cf}$ to the resonance energy of rhodium $(1 \mathrm{eV})$ by measuring the activity of a rhodium foil screened with cadmium covers at various axial locations in a stack of Lucite moderating material. The Lucite plates, whose dimensions are given in Table I, were stacked to form a rectangular parallelepiped $\sim 35.6 \mathrm{~cm}$ square by $34.9 \mathrm{~cm}$ in height. In order to minimize neutron backscattering from the walls and the floor, the stack of Lucite was place on a table in the center of a large room and was covered with thin sheets of cadmium. The neutron source was placed in a fixed position inside the Lucite, while the cadmium-covered rhodium detector was placed in various axial positions as the sample holder insert containing the rhodium foil was moved along the $\mathrm{x}$-axis. Figure 5 shows a cross-section view of the stack and the position of the source and the rhodium detector along the z-axis. It should be mentioned that the rhodium foil was irradiated for $\sim 5 \mathrm{~min}$ in each position, which is $99 \%$ of the saturation activity for this isotope. In addition, once the foil was irradiated, it took $20 \mathrm{~s}$ to retrieve the foil and transport it to the detector room, place it into the aluminum block recess, place the instrument pancake head on the top of it, and start the counting. The E-600 was operated in the integrate mode, and the activity of the foil was measured for $5 \mathrm{~min}$. Prior to the irradiation of the rhodium foil, a background measurement was taken with the E-600 radiation instrument and subtracted from the measured activity of the rhodium foil.

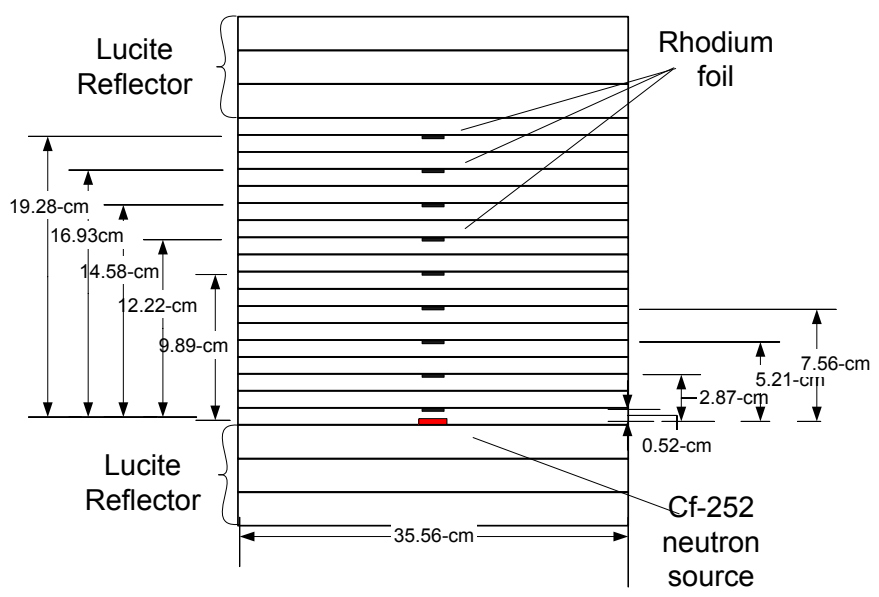

Fig. 5. Cross-section view of the Lucite experiment showing the position where the rhodium foil was placed.

The activity, corrected for background, for the rhodium foil enclosed in cadmium as a function of its z-coordinate, is given in Table II. These results have also been plotted in Fig. 6a. The curve approximates a two group Gaussian distribution. To obtain the best fit, we superimposed two Gaussian groups [Eq. (2)] using a least squares fit to the data given in Table II: 
$q_{1 e V}=1.002 \times 10^{4} * e^{-\frac{z^{2}}{3.321^{2}}}+8.955 \times 10^{3} * e^{-\frac{z^{2}}{8.82^{2}}}$

As Fig. $6 \mathrm{~b}$ illustrates, the two-group Gaussian fit is almost a perfect fit for the experimental data. In addition, the Gaussian fit provides important information regarding the range or distance that fast neutrons from the ${ }^{252} \mathrm{Cf}$ source can travel up to the $1-\mathrm{eV}$ resonance of cadmium in Lucite.

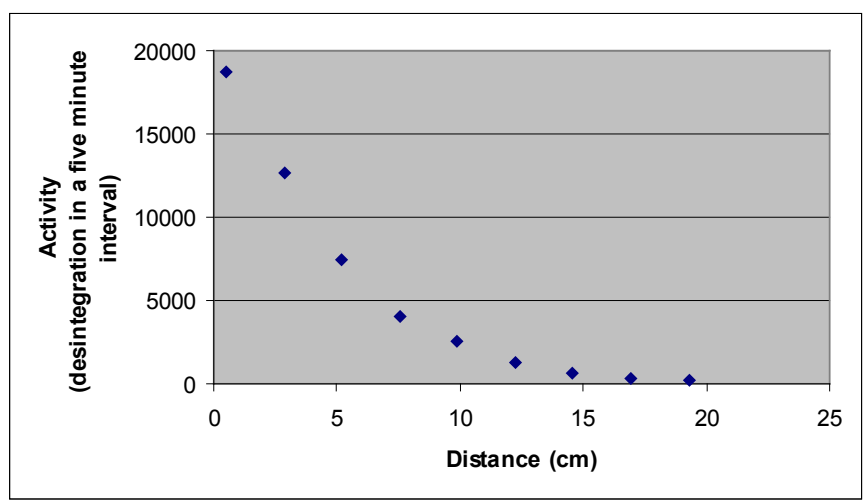

(a)

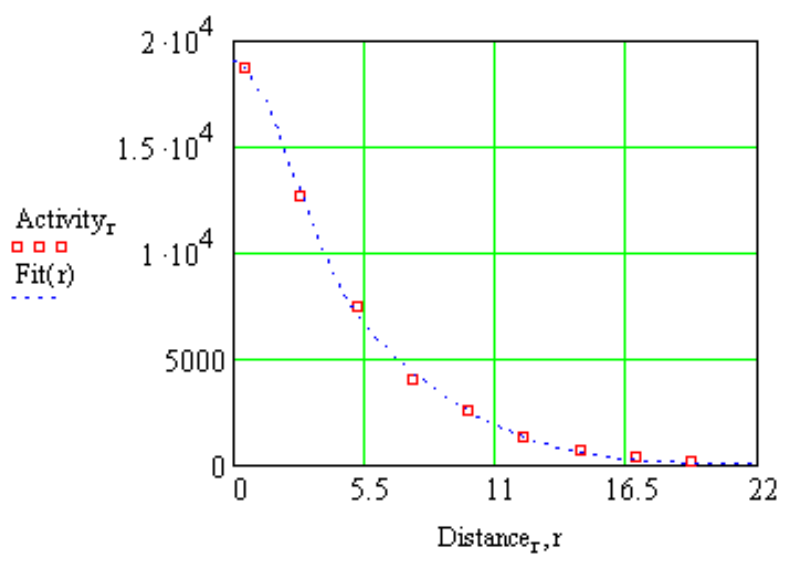

(b)

Fig. 6. (a) Activity of the rhodium foil screened with cadmium as a function of the z-coordinate and (b) least-squares fit of the data presented in (a).

The distance traveled by fast neutrons during the slowdown process is important because it determines the size of a critical system with fissile material. According to Fermi age theory, the slowdown process can be described by Eq. (3):

$$
\Delta q=\frac{\partial q}{\partial t}
$$

where

$\Delta=$ Laplacian operator

$q=$ density of neutrons slowed down to a certain energy

$t=$ parameter known as the Fermi age and dependent on the neutron energy.
TABLE II

DATA COLLECTED FROM THE ACTIVITY OF THE RHODIUM FOIL

\begin{tabular}{|c|c|c|}
\hline $\begin{array}{c}\mathrm{Z} \\
(\mathrm{cm})\end{array}$ & $\begin{array}{l}\text { Activity of } \mathrm{Rh} \\
\text { foil with Cd } \\
\text { covers (counts } \\
\text { in a five minute } \\
\text { interval) }\end{array}$ & $\begin{array}{l}\text { Activity of } \mathrm{Rh} \\
\text { foil with no Cd } \\
\text { covers (counts } \\
\text { in a five minute } \\
\text { interval) }\end{array}$ \\
\hline 0.52 & 18,747 & 208,767 \\
\hline 2.87 & 12,647 & 187,867 \\
\hline 5.21 & 7,467 & 132,568 \\
\hline 7.56 & 4,056 & 95,270 \\
\hline 9.89 & 2,597 & 56,066 \\
\hline 12.22 & 1,327 & 33,267 \\
\hline 14.58 & 642 & 19,769 \\
\hline 16.93 & 335 & 11,527 \\
\hline 19.28 & 203 & 6,547 \\
\hline
\end{tabular}

The dimension of the Fermi age is in square centimeters. Equation (3) resembles the time-dependent diffusion equation and its solution for the neutron point source in an infinite medium with no neutron absorption is given by Eq. (4):

$$
\begin{aligned}
& q=\frac{1}{(4 \pi t)^{3 / 2}} * e^{-\frac{r^{2}}{4 t}}, \\
& r_{o}{ }^{2}=4 t
\end{aligned}
$$

where $r$ is the distance from the source and $r_{0}$ represents the range of the distribution. Both of these equations are derived in Ref. 4. For the case of a rectangular parallelepiped with a point source, the solution of Eq. (3) may be expressed as

$$
q \propto I \propto e^{-z^{2} / 4 t},
$$

where again q represents the density of neutrons slowed down to a certain energy and is proportional to the intensity I or the activity measured, in this case by the rhodium foil, which is also proportional to a Gaussian distribution as a function of the z-coordinate. As seen in Fig. 6 and Eq. (2), the activity as a function of the height in the stack is represented by a twogroup Gaussian or normal distribution. It is clear that the Fermi age model [Eqs. (3) and (4)] does not apply completely to the actual measured distribution. The model predicts one exponential Gaussian distribution instead of the two-group measured distribution. One of the reasons for this discrepancy is that the Fermi age model assumes that the neutron sources in the moderating material are monoenergetic. In reality, the energy of the neutrons emitted by the ${ }^{252} \mathrm{Cf}$ source used in this experiment had a fission energy spectrum similar to that of ${ }^{235} \mathrm{U}$ and was not monoenergetic. This could explain why two- 
groups were needed to get the best fit for the measured distribution. Another reason is that Eqs. (3) and (4) are based on the assumption that the energy loss when neutrons collide with the atoms of moderating material is a continuous function. In reality, the neutron energy is lost in large discrete amounts, especially for collisions with light elements such as hydrogen $[5,6]$. According to Ref. 4, age theory is based on the neutron population from a source diffusing throughout the moderating material. However, for hydrogenous materials, this assumption is not entirely true because the scattering cross section for hydrogen decreases rapidly with increasing neutron energy. Figure 7 illustrates the elastic scattering cross section as a function of neutron energy for Lucite, according to the model created with MCNP [7] and the ENDF/B-VI nuclear reaction database cross-section data. As seen in Fig. 7, the scattering cross section for Lucite is constant between $1 \mathrm{eV}$ and $10 \mathrm{keV}$ but thereafter decreases rapidly with increasing neutron energy, as noted above.

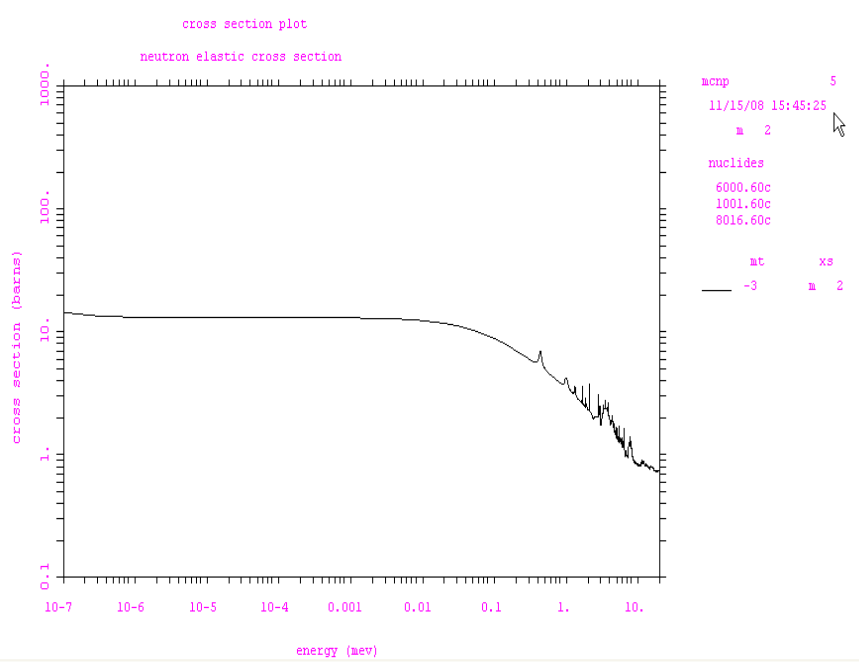

Fig. 7. Scattering cross section fro Lucite based on ENDF/B-VI cross-section data.

Let us considered a fast neutron that is emitted in an infinite hydrogenous medium with energy in the mega-electron-volt range. The neutron undergoes its first collision after moving approximately 1 mean free path, or $1 / \Sigma_{\mathrm{s}}\left(\mathrm{E}_{\mathrm{o}}\right)$, from its starting point. At that time, the neutron loses a certain amount of energy. Because of this loss of energy, the scattering cross section for the second collision is much larger, as seen in Fig. 7. Since the mean free path is inversely proportional to the scattering cross section, the second mean free path is considerably smaller than the first one. Therefore, the second neutron collision occurs near the location of the first one. This process continues with the result that neutrons in hydrogenous medium migrate short distances from the point where the first scattering event occurs. In other words, high-energy neutrons do not diffuse as expected, according to Fermi age theory, when the neutrons are in a hydrogenous environment. Nonetheless, Fermi age theory provides experimental data for hydrogenous materials that can be compared to those of other materials in which the diffusion process is as expected.

Equation (2) provides two ranges for the fast neutron population to slow down to $1 \mathrm{eV}$. These two ranges are 3.321 and $8.82 \mathrm{~cm}$, respectively. The two terms in Eq. (2) represent two fast-energy groups of neutrons diffusing with different ranges. We can use Eq. (6) to estimate the range for thermal neutrons in Lucite, based on the range calculated for the rhodium foil ${ }^{2}$ at $1 \mathrm{eV}$ :

$$
r_{o}^{2}(\text { thermal })=20.6 * \lambda^{2} * \log _{10} \frac{E_{R h}}{E_{t h}}+r_{o}^{2}(R h),
$$

where

$$
\begin{array}{ll}
\lambda & =\text { mean free path for Lucite } \\
\mathrm{E}_{\mathrm{Rh}}= & \text { absorption resonance energy of rhodium due to } \\
& \text { the encapsulation with cadmium. } \\
\mathrm{E}_{\mathrm{th}} \quad=\text { energy of thermal neutrons }(0.025 \mathrm{eV}) .
\end{array}
$$

The value of $r_{o}{ }^{2}$ for rhodium is the weighed average of the two ranges found with Eq. 2. Thus, if $\lambda$ is assumed to be $0.32 \mathrm{~cm}$, the average range of the fast neutrons slowing down to thermal energies in Lucite is $6.3 \mathrm{~cm}$. Again, this method does not take into account the chemical bonds of the Lucite molecule. The mean free path for Lucite was calculated on the basis of the experimental total cross section data at thermal energies provided by F. Tovesson. It is interesting to note that Fermi performed similar experiments with other types of materials. One of these materials that became very important for the development of nuclear reactor engineering was graphite. Fermi measured the average range of fast neutrons slowing down to thermal energies for graphite. The experiment yielded a value of $37-\mathrm{cm}$, which compares quite well with the value that we obtained for Lucite.

\section{THERMAL DIFFUSION PROCESS}

Once the energy of fast neutrons has been reduced to thermal energies, the thermal neutrons continue to diffuse throughout the moderating material without significant loss of energy until they are finally absorbed. The range or distance that the thermal neutron can travel depends on the absorption cross section of the moderating material. The behavior of thermal neutrons in moderating materials can be modeled with the help of Eq. 7:

$$
\frac{\partial n}{\partial t}=\frac{\lambda \mathrm{v}}{3} \Delta n-\frac{1}{\tau} n+q
$$

where the term on the left side of the equal sign represents the rate of change of the thermal neutrons in the system, and the first term on the right side represents the leakage or diffusion of thermal neutrons where $\lambda v / 3$ is the diffusion coefficient. The second term on the right side represents the absorption of thermal neutrons, and the third term represents the production of thermal neutrons due to the slowing down process described in the previous section. For the steady state case, using the 
relationship for the mean life $\tau=\lambda N / \mathrm{v}$ for thermal neutrons, we obtain the following equation:

$$
n=\frac{\lambda^{2} N}{3} \Delta n+q \frac{\lambda N}{\mathrm{~V}}
$$

where $N\left(\mathrm{~N}=\sigma_{\text {scat }} / \sigma_{\text {abs }}\right)$ is the number of collisions that thermal neutrons undergo before being absorbed, $\mathrm{v}$ is the average velocity of thermal neutrons, and $\Delta n$ is the Laplacian of $n$. All the other variables have been previously defined.

In order to determine the absorption cross section in Lucite, we performed another measurement of the neutron distribution at the same positions along the z-axis as in the previous experiment. This time, however, the rhodium foil was not screened with cadmium covers. The rhodium foil was again irradiated for 5 minutes at each location and counted with the GM counter for 5 minutes. As in the previous experiment, the experiment was covered with a thin sheet of cadmium to reduce the room return component into the experiment. The results from these measurements are listed in Table II. It should be mentioned that the activity in the rhodium foil when the foil is screened with cadmium covers is due entirely to neutrons with energies greater than the cadmium cutoff of 1 $\mathrm{eV}$. On the other hand, the activation of the rhodium foil with no cadmium covers is due not only to the neutrons with energies above the cadmium cutoff of $1 \mathrm{eV}$ but also to neutrons with thermal energies. If we were to subtract these two activities, we would end up with the contributions of thermal neutrons only, and Eq. 8 would be transformed into the following expression:

$$
n_{2}-n_{1}=\frac{\lambda^{2} N}{3} \Delta\left(n_{2}-n_{1}\right)
$$

The boundary conditions at which $\mathrm{n}_{2}-\mathrm{n}_{1}=0$ are simply the dimensions of the stack plus the extrapolation distances given by $\lambda /(3)^{1 / 2}$. The solution to Eq. 9 is

$n_{2}-n_{1}=\sum_{j, k=1}^{\infty} A_{j, k} \sin \frac{\pi j x}{a} \sin \frac{\pi k y}{a}\left[e^{-\alpha_{j, k} z}-e^{-2 \alpha_{j, k} b} e^{\alpha_{j, k^{z}}}\right]$,

where

$$
\alpha_{j, k}^{2}=\frac{3}{\lambda^{2} N}+\frac{\pi^{2}}{a^{2}}\left(j^{2}+k^{2}\right)
$$

and $a$ represents the dimension of the actual side of the Lucite, including the extrapolation distance. In our experiment, $a$ is equal to $35.93 \mathrm{~cm}, b$ is length in the axial direction from the neutron source and is equal to $27.99-\mathrm{cm}$, and $j$ and $k$ are equal to 1, which represents the first harmonic or the fundamental mode. To determine the absorption cross section, we needed to evaluate the difference of the data presented in Table II. These data have been plotted in Fig. 8 , and the $\alpha_{1,1}$ has been found by least squares fitting. As shown in Fig. 8, the $\mathrm{R}^{2}$ value of the fit is almost 1 , which indicates a very good fit.
Based on these measurements, the value of $\alpha_{1,1}$ from Fig. 8 is 0.224 . Using Eq. 11, we can determine the number of collisions that thermal neutrons undergo before being captured. This number is 828 collisions. The thermal neutron absorption cross section is found by solving for $\sigma_{\mathrm{abs}}$ in Eq. 12 .

$$
\mathrm{N}=\sigma_{\text {scatt }} / \sigma_{\text {abs }}
$$

The value used for $\sigma_{\text {scatt }}$ in Eq. 12, which is almost entirely $\sigma_{\text {tot }}$, is 433 barns and is a measured value obtained in a neutron transmission measurement performed at the Los Alamos Neutron Science Center (LANSCE). A Lucite sample was irradiated with the moderated white neutron beam at LANSCE, and the time of flight method was used to determine the incident neutron energy. The neutron flux was monitored with fission ionization chambers using sample-in/sample-out measurements to deduce the total cross section as a function of incident neutron energy, as shown in Fig. 9. The same experimental set up and method were used for other transmission measurements, and are described in Ref. 8.

$$
\mathrm{R} 2=0.998, e^{-0.224 z}-e^{-0.224 * 55.99 z} * e^{-0.224 z}
$$

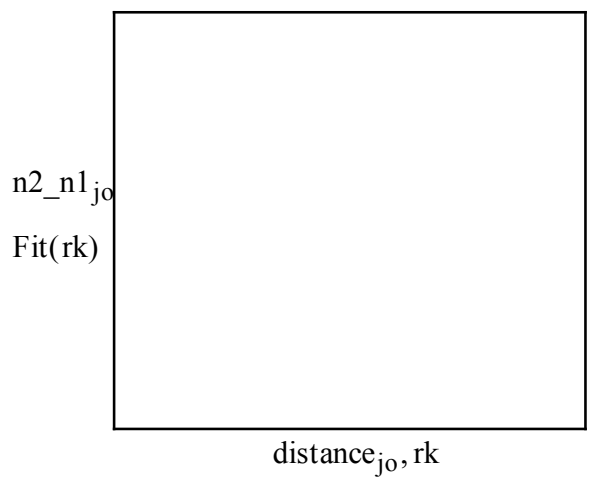

Fig. 8. Plot of $\mathrm{n}_{2}-\mathrm{n}_{1}$ as a function of distance from the neutron source and the fit of the data.

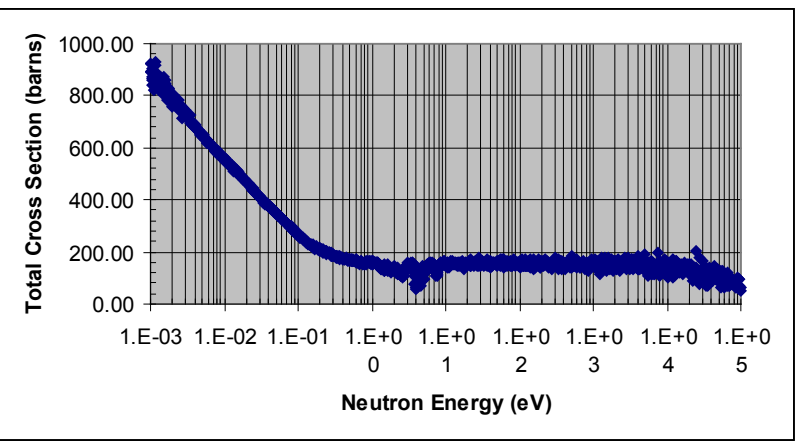

Fig. 9. Total neutron cross section as a function of neutron energy for Lucite.

The value found for the thermal absorption cross section in Lucite is 0.52 barns, which over predicts the ENDF/B-VI value of 0.27 barns. This could explain why MCNP with 
ENDF/B-VI cross section data calculates $1 \%$ to $2 \%$ higher multiplication factors, $\mathrm{k}_{\mathrm{eff}}$, for certain uranium/Lucite critical configurations.

\section{CONCLUSIONS}

Several experiments were performed to investigate the neutron diffusion processes in Lucite and to determine its thermal neutron cross section. The experiments were based on previous experiments that Enrico Fermi performed in the $1930 \mathrm{~s}$ and $1940 \mathrm{~s}$ to investigate the neutron absorption properties of various materials. An average value of $6.3 \mathrm{~cm}$ was found for the range of fast neutrons slowing down to thermal energies. The experiments yielded a thermal absorption cross section of 0.52 barns.

\section{ACKNOWLEDGMENT}

The authors would like to acknowledge Erik Sorensen and his operating staff, Christopher Romero and Benjamin Montoya, for providing the mechanical engineering design and support needed for these experiments.

\section{REFERENCES}

[1] E. Fermi, et al., "On the Motion of Neutrons in Hydrogenous Substances," The Collected Papers of Enrico Fermi, Vol. !. pp. 9801016, University of Chicago Press, Chicago (1962).
[2] H. L. Anderson and E. Fermi, "Production and Absorption of Slow Neutrons by Carbon," The Collected Papers of Enrico Fermi, Vol. !. pp. 980-1016, University of Chicago Press, Chicago (1962).

[3] P. R. Steinmeyer, "G-M Pancake Detectors: Everything You 've Wanted to Know (But Afraid to Ask)," RSO Magazine, 10, 5, 7 (2005).

[4] J. R. Lamarsh, Nuclear Reactor Theor, pp. 187-198, Addison-Wesley, Reading, Massachusetts (1972).

[5] D. J. Hughes, Pile Neutron Research, pp. 123-127 (Addison-Wesley, Reading, Massachusetts (1953)

[6] E. Fermi, "Elementary Theory of the Pile," LAMS-427, Los Alamos Scientific Laboratory (1946).

[7] “MCNP- A General Monte Carlo N-Particle Transport Code," Version 4C, J. F. Briesmeister, Ed., LA-12625-M, Los Alamos National Laboratory (Mar. 2000).

[8] G. Muhrer, T. Hill, F. Tovesson, and E. Pitcher, "Comparison of the Measured and Calculated Total Thermal Neutron Cross-Section of Pb," Nucl. Instrum. Methods Phys. Res. A, 572, 866 (2007). 